\title{
Primary fallopian tube cancer in the setting of endometriosis
}

\author{
Deeksha Pandey*, Varun Bhalla, Anushri Mittal, Jyothi Shetty
}

Department of Obstetrics \& Gynaecology, KMC Manipal, Manipal University, Karnataka, India

Received: 10 July 2015

Revised: 30 August 2015

Accepted: 02 September 2015

\author{
*Correspondence: \\ Dr. Deeksha Pandey, \\ E-mail: deekshiiiobg@gmail.com
}

Copyright: (c) the author(s), publisher and licensee Medip Academy. This is an open-access article distributed under the terms of the Creative Commons Attribution Non-Commercial License, which permits unrestricted non-commercial use, distribution, and reproduction in any medium, provided the original work is properly cited.

\begin{abstract}
We report the first case of PFTC arising in the setting of pelvic endometriosis. A 45 year old nulliparous lady, menopausal lady presented with pelvic mass. She had history of pelvic endometriosis. MRI findings were suggestive of ovarian malignancy. Histological analysis of specimen obtained following staging laparotomy proved it to be a case of bilateral PFTC. She received 6 cycles of adjuvant chemotherapy. This case opens a new door to think or investigate if fallopian tube endometrioma behaves as a more aggressive premalignant lesion. This case also poses questions whether it is important to differentiate an endometrioma arising from the ovary to one which is arising from a fallopian tube. Once identified can fallopian tube endometrioma also be managed medically or should it be always removed surgically?
\end{abstract}

Keywords: Pelvic endometriosis, Ovarian malignancy

\section{INTRODUCTION}

Primary fallopian tube cancer (PFTC) is a rare gynaecological malignancy that is easy to miss, and presents as a diagnosis, management and prognosis dilemma. $^{1,2}$ We report the first case of PFTC arising in the setting of pelvic endometriosis.

\section{CASE REPORT}

A 45 year old nulliparous lady, menopausal for 14 months presented to us with complaints of vaginal spotting. On evaluation of infertility she was found to have pelvic endometriosis 10 years ago. She underwent enucleation of endometriotic cyst 5 years ago, elsewhere. Surgical records were not available.

When she presented to us her vital parameters were in normal range. Abdominal examination, revealed midline vertical scar of previous surgery. Abdomen was soft, nontender with no palpable mass. On speculum examination the cervix was normal in size and healthy with no discharge. On vaginal examination uterus could not be felt separately. A pelvic mass around $6 \mathrm{~cm} \mathrm{X} 6 \mathrm{~cm}$ with variegated consistency and restricted mobility was felt.

Ultrasonography showed heterogeneous lesions measuring $8.2 \times 5.1 \mathrm{~cm}$ with anechoic and solid components in the pelvis extending up to the Pouch of Douglas and left adnexa. The lesion was abutting the posterior uterine wall and lower uterine segment with indistinct fat planes. Right and left ovary could not be distinguished from the lesion. Uterus was normal in size and there was no evidence of free fluid. Magnetic resonance imaging (MRI) revealed well-defined heterogeneous, mixed cystic and solid lesions in both the adnexa (left $-9.8 \times 7 \times 5.8$; right $-4.3 \times 2.2 \times 2.8$ ) which couldn't be visualized separately from the ovaries. The left lesion showed fluid-debris level indicative of an associated hemorrhage due to endometriosis (figure 1). Para-aortic lymphadenopathy was detected suggesting the possibility of ovarian malignancy. CA-125 was $16 \mathrm{U} / \mathrm{ml}$. 
With a diagnosis of ovarian malignancy based of MRI findings, patient underwent staging laparotomy. Peroperatively minimal ascites was noted. Both the ovaries were enlarged with solid-cystic areas and were adherent to the uterus forming a conglomerate mass $10 \times 10 \mathrm{~cm}$. Pouch of Douglas was obliterated due to adhesions present between the mass and sigmoid colon. Omentum and appendix appeared grossly normal. Uterus along with the adnexa and cervix was removed. Infracolic omentectomy, appendectomy, and bilateral pelvic lymphadinectomy (external and internal iliac nodes and obturator nodes) were also performed. Biopsies from culde-sac peritoneum, lateral pelvic walls, and bilateral paracolic peritoneum were taken. Whole specimen was subjected to histological analysis. Histopathology diagnosed it to be - serous papillary adenocarcinoma of both fallopian tubes (Figure 2) with bilateral metastasis to right internal and external iliac lymph nodes (T2aN1M0, clinical stage 2).

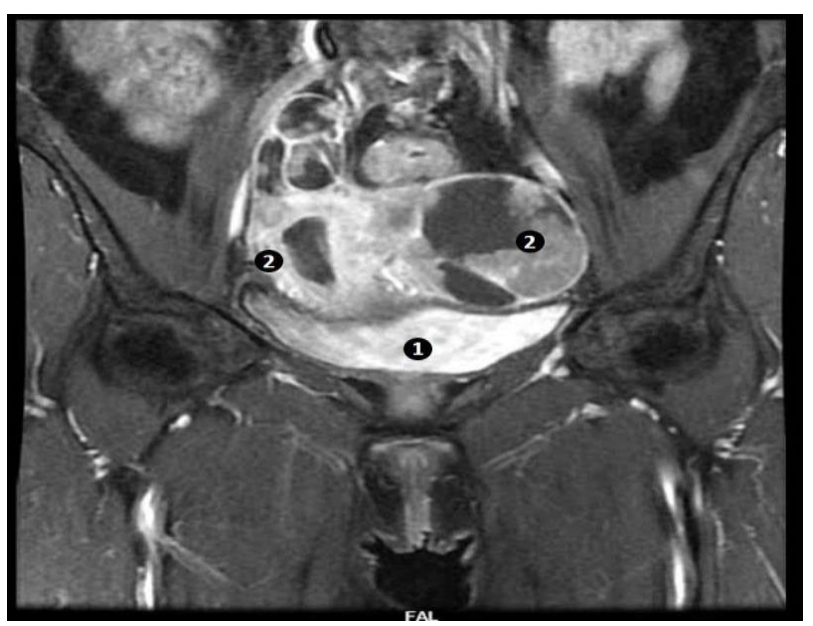

Figure 1: MRI T1 coronal image showing well-defined heterogeneous, mixed cystic and solid lesions in both the adnexa which couldn't be visualized separately from the ovaries. The left lesion showed fluid-debris level indicative of an associated hemorrhage due to endometriosis. 1) Bladder 2) Adnexal mass.

Patient recovered well post operatively. She received six cycles of adjuvant chemotherapy (Paclitaxel and Carboplatin). The patient was asymptomatic till 11 months post-surgery, when she presented with breathlessness of sudden onset. There was associated history of weight loss and loss of appetite. She was thoroughly evaluated for the same. Fine needle aspiration cytology (FNAC) of left mediastinal lymph nodes showed poorly differentiated malignant cells. Pleural fluid cytology was positive for malignant cells with features suggestive of adenocarcinoma. Bronchial brush cytology was negative for features of malignancy. This led to a diagnosis of bilateral serous-papillary adenocarcinoma of the fallopian tubes with secondary's to the lungs and malignant pleural effusion. Thus she is started on palliative chemotherapy.

\section{DISCUSSION}

We report here a case of bilateral PFTC which was diagnosed only after the histological examination following staging laparotomy for ovarian malignancy (pre-operative diagnosis). To our knowledge this is the first case of PFTC in a setting of endometriosis, in English literature.

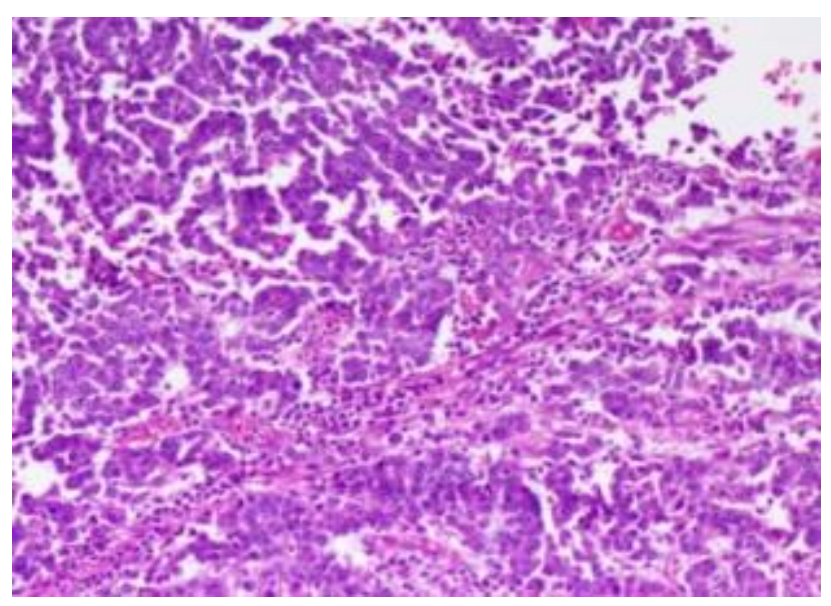

Figure 2: Histopathological examination of both the tubes revealed malignant cells arranged in complex branching papillae.

PFTC is a rare cancer accounting for less than $2 \%$ of female genital malignancies. However this might be due to underestimation as this cancer many a times gets misidentified as epithelial ovarian cancer (EOC) during initial evaluation. ${ }^{3}$ Involvement of both the tubes has been reported in around $10 \%-30 \%$ of cases in various studies.

Recent molecular and clinicopathologic studies have failed to support the existing hypothesis that EOC arises from the ovarian surface epithelium. The new model of ovarian carcinogenesis proposes that fallopian tube epithelium implants on the ovary to give rise to ovarian malignancy. ${ }^{4}$ As borderline ovarian tumors, even endometriosis has been hypothesized as a premalignant lesion. ${ }^{4}$ More recently even ovarian endometriosis has been postulated to be originating from the fallopian tubes. Up to $60 \%$ of the ovarian endometriosis may be derived from the fallopian tube. ${ }^{5}$ As in the present case patient had a long standing endometrioma which progressed to PFTC. This case opens a new door to think or investigate if fallopian tube endometrioma behaves as an aggressive premalignant lesion. This case also poses questions whether it is important to differentiate an endometrioma arising from the ovary to one which is arising from a fallopian tube? Once identified can fallopian tube endometrioma also be managed medically or should it be always removed surgically?

This case reported here had a history of infertility. Infertility has been found to be associated with five folds higher incidence of bilateral fallopian tube cancer. ${ }^{6}$ 
However, the prognosis is reported better in nulliparous women. $^{7}$

Clinical features of fallopian tube cancer are non-specific. Latzko's triad of symptoms (intermittent profuse serosanguinous vaginal discharge, colicky pain relieved by discharge, and abdominal or pelvic mass) has been reported only in $15 \%$ of cases. ${ }^{8}$ Our patient has none of these characteristic features.

Studies have shown that MRI can identify the characteristic features of PFTC and differentiate PFTC from EOC. PFTC often appears as a small-sized solid mass, with a sausage-like shape, homogeneous signal, mild or moderate enhancement, hydrosalpinx, or intrauterine fluid accumulation. ${ }^{9}$ In the present case however the features pointed towards EOC, without any evidence of primary in fallopian tube.

Published literature says that CA-125 antigen is often expressed by PFTC10 and most of the patients have elevated pre-treatment serum CA-125 levels which correlate well to the disease stage. ${ }^{3}$ However in the present case serum CA-125 was only $16 \mathrm{U} / \mathrm{ml}$ which did not correlate with the disease stage.

Management of PFTC is similar to EOC,11 though the prognosis in terms of survival is reported to be better as compared to ovarian, peritoneal, and uterine cancer. ${ }^{12}$

\section{CONCLUSION}

In this study, we presented a case of ovarian leiomyosarcoma with multiple fibroid uterus with normal serum tumour marker level. We found that even in advanced cases, surgical debulking gives good symptomatic relief, so appropriate surgical treatment should not be delayed solely on the basis of normal tumor marker levels. Because of its poor prognosis, early diagnosis and prompt surgical removal are important for patients with ovarian leiomyosarcoma for improvement of symptoms and in survival.

Funding: No funding sources

Conflict of interest: None declared

Ethical approval: Not required

\section{REFERENCES}

1. Horng HC, Teng SW, Huang BS, Sun HD, Yen MS, Wang $\mathrm{PH}$, et al. Primary fallopian tube cancer: Domestic data and up-to-date review. Taiwanese journal of obstetrics \& gynecology. 2014;53(3):28792.
2. Horng HC, Teng SW, Lai CR, Chang WH, Chang $\mathrm{YH}$, Yen MS, et al. Prognostic factors of primary fallopian tube cancer in a single institute in Taiwan. International journal of gynaecology and obstetrics: the official organ of the International Federation of Gynaecology and Obstetrics. 2014;127(1):77-81.

3. Pectasides D, Pectasides E, Economopoulos T. Fallopian tube carcinoma: a review. The oncologist. 2006;11(8):902-12.

4. Kuhn E, Kurman RJ, Shih IM. Ovarian Cancer Is an Imported Disease: Fact or Fiction? Current obstetrics and gynecology reports. 2012 Mar;1(1):1-9. PubMed PMID: 22506137.

5. Yuan Z, Wang L, Wang Y, Zhang T, Li L, Cragun $\mathrm{JM}$, et al. Tubal origin of ovarian endometriosis. Modern pathology : an official journal of the United States and Canadian Academy of Pathology, Inc. 2014;27(8):1154-62.

6. Meng ML, Gan G, Scheng S, Bao QC, Jung ZA. Diagnosis of primary adenocarcinoma of the fallopian tube. Journal of cancer research and clinical oncology. 1985;110(2):136-40.

7. Hanton EM, Malkasian GD, Dahlin DC, Pratt JH. Primary carcinoma of the fallopian tube. American journal of obstetrics and gynecology. 1966;94(6):832-9.

8. Ajithkumar TV, Minimole AL, John MM, Ashokkumar OS. Primary fallopian tube carcinoma. Obstetrical \& gynecological survey. 2005;60(4):24752.

9. Ma FH, Cai SQ, Qiang JW, Zhao SH, Zhang GF, Rao YM. MRI for differentiating primary fallopian tube carcinoma from epithelial ovarian cancer. Journal of magnetic resonance imaging: JMRI, 2014.

10. Puls LE, Davey DD, DePriest PD, Gallion HH, van Nagell JR, Jr., Hunter JE, et al. Immunohistochemical staining for CA-125 in fallopian tube carcinomas. Gynecologic oncology. 1993;48(3):360-3.

11. Zeppernick F, Meinhold-Heerlein I. The new FIGO staging system for ovarian, fallopian tube, and primary peritoneal cancer. Archives of gynecology and obstetrics, 2014.

12. Usach I, Blansit K, Chen LM, Ueda S, Brooks R, Kapp DS, et al. Survival differences in women with serous tubal, ovarian, peritoneal, and uterine carcinomas. American journal of obstetrics and gynecology. 2014 Aug 18. PubMed PMID: 25149685. Epub 2014/08/26. Eng. Enzinger FM, Weiss SW. Soft tissue sarcoma. The CV Mosby Co. 1988;2:402.

Cite this article as: Pandey D, Bhalla V, Mittal A, Shetty J. Primary fallopian tube cancer in the setting of Endometriosis. Int J Reprod Contracept Obstet Gynecol 2015;4:1571-3. 